\title{
Framework for the evaluation of new tests for tuberculosis infection
}

\author{
Yohhei Hamada $\mathbb{1}^{1,2}$, Saskia den Boon ${ }^{3}$, Daniela Maria Cirillo $\mathbb{\circledR}^{4}$, Adam Penn-Nicholson ${ }^{5}$, \\ Morten Ruhwald ${ }^{5}$, Dick Menzies ${ }^{6}$, Olivia Oxlade ${ }^{6}$, Dennis Falzon ${ }^{3}$, Avinash Kanchar $^{3}$, Alexei Korobitsyn $^{3}$, \\ Matteo Zignol ${ }^{3}$, Alberto Matteelli ${ }^{7}$ and Tereza Kasaeva ${ }^{3}$
}

\begin{abstract}
${ }^{1}$ Research Institute of Tuberculosis, Japan Anti-Tuberculosis Association, Tokyo, Japan. ${ }^{2}$ University College London, London, UK. ${ }^{3}$ Global TB Programme, World Health Organization, Geneva, Switzerland. ${ }^{4}$ IRCCS San Raffaele Scientific Institute, Milan, Italy. ${ }^{5}$ Foundation for Innovative New Diagnostics (FIND), Geneva, Switzerland. ${ }^{6}$ McGill International TB Centre, Montreal, QC, Canada. ${ }^{7}$ Collaborating Centre for TB/HIV Co-infection and TB Elimination, Dept of Infectious and Tropical Diseases, University of Brescia, Brescia, Italy.
\end{abstract}

Corresponding author: Yohhei Hamada (y.hamada@ucl.ac.uk)

Shareable abstract (@ERSpublications)

The evaluation of new, improved tests for tuberculosis infection should be expedited using standard study protocols to accelerate adoption into policy and subsequent scale-up. A framework for such evaluation is described. https://bit.ly/38ChJbe

Cite this article as: Hamada Y, den Boon S, Cirillo DM, et al. Framework for the evaluation of new tests for tuberculosis infection. Eur Respir J 2021; 58: 2004078 [DOI: 10.1183/13993003.04078-2020].

Copyright $\odot$ The authors 2021.

This version is distributed under the terms of the Creative Commons Attribution Licence 4.0.

This article has supplementary material available from erj. ersjournals.com

Received: 4 Nov 2020 Accepted: 2 Jan 2021

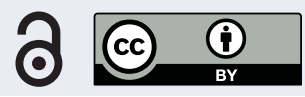

\section{Abstract}

The scale-up of tuberculosis (TB) preventive treatment (TPT) must be accelerated to achieve the targets set by the United Nations High-level Meeting on TB and the End TB Strategy. The scale-up of effective TPT is hampered by concerns about operational challenges to implement the existing tests for TB infection. New simpler tests could facilitate the scale-up of testing for TB infection. We present a framework for evaluation of new immunodiagnostic tests for the detection of TB infection, with an aim to facilitate their standardised evaluation and accelerate adoption into global and national policies and subsequent scale-up. The framework describes the principles to be considered when evaluating new tests for TB infection and provides guidance to manufacturers, researchers, regulators and other users on study designs, populations, reference standards, sample size calculation and data analysis and it is also aligned with the Global Strategy for TB Research and Innovation adopted by the World Health Assembly in 2020. In addition, we briefly describe technical issues that should be considered when evaluating new tests, including the safety for skin tests, costs incurred by patients and the health system, and operational characteristics.

\section{Introduction}

Treatment of tuberculosis (TB) infection, also known as tuberculosis preventive treatment (TPT), is a critical component needed to achieve the ambitious targets of the End TB Strategy 2016-2035 [1]. Management of TB infection is also critical to pursue TB elimination [2]. Without the prospect that a new, efficacious and safe TB vaccine can be developed and scaled-up worldwide in the foreseeable future, continued measures to expand the provision of effective TPT remain of critical importance. Furthermore, at the first United Nations High-level Meeting on TB in 2018, member states committed to provide TPT to $\geqslant 30$ million people in 2018-2022: 6 million people living with HIV (PLHIV), 4 million children aged $<5$ years who are household contacts of people with TB and 20 million other household contacts [3]. Progress in expanding TPT coverage among contacts of TB patients has been very limited up to now, with approximately half a million contacts in 2019 [4]. There is no gold standard method for diagnosing TB infection [5]. The World Health Organization (WHO) currently recommends a tuberculin skin test (TST) or an interferon- $\gamma$ release assay (IGRA) to test for TB infection [6]. The tests are helpful to identify people at higher risk of developing TB disease and who could benefit from TPT, because in most published studies such risk is higher in people who test positive for TB infection than in those who test negative [7]. However, current tests for TB infection have limited value in predicting the risk of progression from infection to active TB disease [8]. 
The development and evaluation of tests characterised by higher prediction capacity (called tests of progression) is a high priority for research. In 2018, the WHO published a guidance document on the characteristics of such tests [9]. According to the target product profile defined by the WHO, optimal sensitivity and specificity of such tests for predicting development of TB disease are $\geqslant 90 \%[9,10]$, much higher than those of the currently available TST or IGRA [11]. Until better tests become available for use under field conditions, existing tests for TB infection, including TST and IGRA, remain the standard tests of choice.

Tests for TB infection are not required before starting TPT in people from high-priority groups such as PLHIV and household contacts aged $<5$ years in high TB burden countries (figure 1) [6]. For people from other at-risk populations, tests for TB infection are recommended to identify those who would benefit most from treatment and to avoid unnecessary treatment, which carries a risk of adverse events (figure 1). However, implementation of tests for TB infection is fraught with difficulties, including short supply of quality-assured purified protein derivative, the need for training to perform and read TST, inadequate laboratory set-up to undertake IGRA testing in decentralised settings and high costs (IGRA). This calls for new tests with better operational characteristics.

New versions of TST and IGRA are already on the market or in the pipeline, all using ESAT6 and CFP10 antigens. Diaskintest (Generium, Moscow, Russia) [12] and ESAT6-CFP10 (Anhui Zhifei Longcom Biopharmaceutical Co. Ltd, Anhui, China) [13], both of which are IGRA-like skin tests for TB infection, are commercially available, and a new IGRA-like skin test, C-Tb (Serum Institute of India, Pune, India) $[14,15]$, has recently been developed. QIAGEN (Venlo, the Netherlands), the manufacturer of the IGRA test QuantiFERON-TB Gold Plus, and SD Biosensor (Suwon-Si, Republic of Korea) have both developed simplified versions of IGRA that can operate with less sophisticated laboratory support (M. Ruhwald; "Future tests for TB infection", presented at the 51st Union World Conference on Lung Health; 20-24 October 2020).

Evaluation of tests for TB infection is not straightforward, due to the lack of a reference standard. Here we present a framework for evaluation of new immunodiagnostic tests for the detection of TB infection, with an aim to facilitate their standardised evaluation and accelerate adoption into global and national policy and subsequent scale-up. The document is primarily intended to guide the work of manufacturers of diagnostics, researchers, funders, regulators, TB programme coordinators, civil society and other stakeholders. The framework was developed by a WHO-convened technical expert group with inputs from reviewers with expertise in this field [16].

The focus of this framework is on the evaluation of diagnostic performance of tests for TB infection. Additionally, we briefly outline technical issues that should be considered when evaluating new tests for TB infection, evaluation of safety for skin tests, costs incurred by patients and the health system and operational characteristics.

\section{Framework for evaluation of new tests for TB infection}

The lack of an adequate gold standard complicates the estimation of sensitivity and specificity, the standard approach to assess the performance of new diagnostic tests. Surrogate reference standards are therefore proposed. A hierarchy of reference standards was developed when the WHO reviewed evidence on the use of IGRA (figure 2) [11].

Reference standards at higher levels of hierarchy should provide stronger evidence for a test's accuracy to identify people who would benefit most from TPT. New tests for TB infection currently in the pipeline are based on similar concepts to TST and IGRA; that is, eliciting an immune response to Mycobacterium tuberculosis-specific antigens either in vivo (size of skin induration) or in vitro (magnitude of cytokine release) [17]. Therefore, they are not expected to offer significant advantage in predicting risk of progression to TB disease. In general, the predictive performance of any new test for TB infection should not be inferior to current technology. Given the challenges to measure predictive value (see later), studies comparing sensitivity, specificity and concordance between new and current tests may be the best feasible option. In this case, a new test for TB infection could demonstrate noninferior sensitivity and specificity based on a pre-specified margin or concordance compared with at least one of the currently available tests endorsed by the WHO as rule-in tests for TPT. Because there is mounting evidence that IGRA has higher specificity and possibly higher sensitivity than TST $[18,19]$, IGRA should be preferred as a comparator in new trials $[14,20]$. Any new tests intended to achieve significant improvement in predictive performance should follow the evaluation framework for tests for predicting progression to TB disease [9, 10]. 


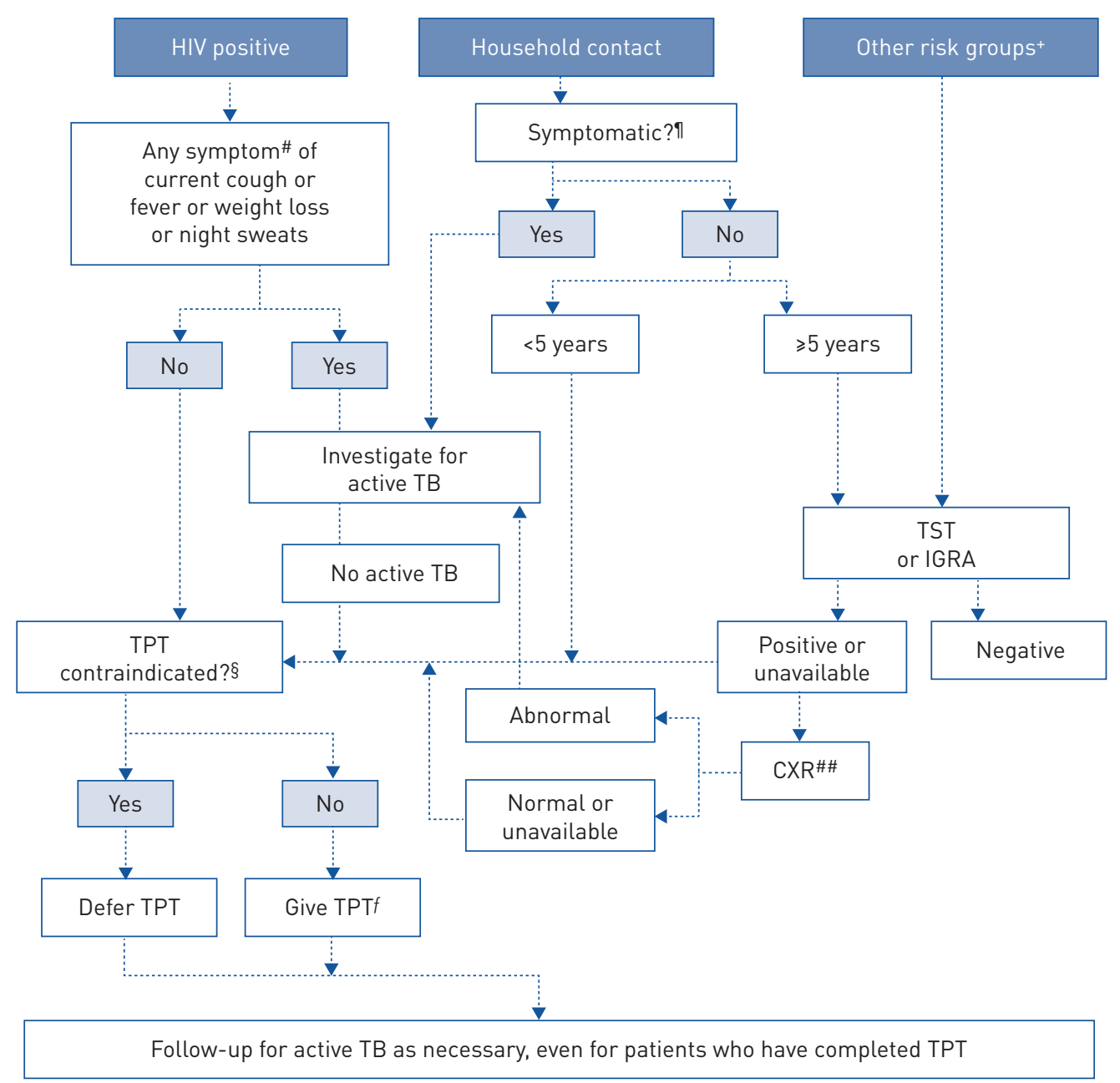

FIGURE 1 Algorithm for testing and treating tuberculosis (TB) infection in different groups considered to be at risk. ": if aged $<10$ years, any one of current cough or fever or history of contact with a person diagnosed with TB or reported weight loss or confirmed weight loss $>5 \%$ since last visit or growth curve flattening or weight for age $<-2$ z-scores. Asymptomatic infants with HIV aged $<1$ year are only treated for TB infection if they are household contacts of TB. Tuberculin skin test (TST) or an interferon- $\gamma$ release assay (IGRA) may identify people living with HIV (PLHIV) who will benefit most from preventive treatment. Chest radiography (CXR) may be used in PLHIV on antiretroviral therapy, before starting treatment of TB infection. ": any one of cough or fever or night sweats or haemoptysis or weight loss or chest pain or shortness of breath or fatigue. In children aged $<5$ years, they should also be free of anorexia, failure to thrive, not eating well, decreased activity or playfulness to be considered asymptomatic. ${ }^{+}$: including silicosis, dialysis, anti-tumour necrosis factor agent treatment, preparation for transplantation or other risks in national guidelines. ${ }^{\text {\& }}$ : including acute or chronic hepatitis, peripheral neuropathy (if isoniazid is used) or regular and heavy alcohol consumption. Pregnancy or a previous history of TB are not contraindications. ${ }^{f}$ : regimen chosen based on considerations of age, strain (drug susceptible or otherwise), risk of toxicity, availability and preferences. ${ }^{\# \#}$ : CXR may have been carried out earlier on as part of intensified case finding. TPT: TB preventive treatment. Reproduced and modified from [6] with permission.

\section{Study design and population}

\section{Study design 1: predictive performance}

A prospective longitudinal study measuring predictive value is the most appropriate method to compare performance of tests for TB infection, and could thus inform future policy on the use of new tests (figure 3). The WHO recommendation to use either TST or IGRA to test for TB infection is based on a review of this type of study $[6,21]$. Using this study design, people with positive or negative tests for TB infection are screened for TB disease; those who are free of TB disease are then followed for $\geqslant 12$ months for development of TB disease. Follow-up of $\geqslant 12$ months is recommended to ensure a sufficient length of time for 


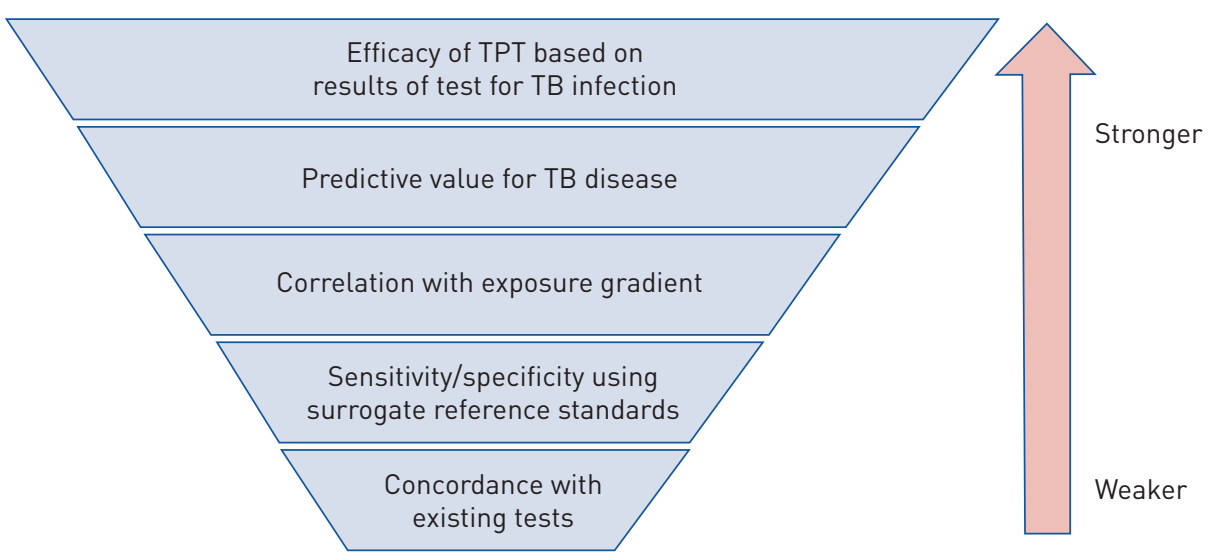

FIGURE 2 Hierarchy of reference standards used to assess the evidence base for tests for tuberculosis (TB) infection. TPT: TB preventive treatment. Reproduced and modified from [16] with permission.

progression to occur. The major ethical issue with this design is that people at risk of TB with a positive test for TB infection should be started on TPT, which interferes with the primary end-points such as TB incidence or mortality. Although TB incidence can be measured in people who test positive for TB infection but who do not take treatment, this will reduce the study power and may also introduce bias given that people opting out may differ in characteristics associated with end-points from those who do take treatment. Moreover, the study design may preclude masking of the study subjects (e.g. comparing a new TST to IGRA). In addition, people who do not follow the recommendations from the provider are more likely to discontinue, which may seriously jeopardise the integrity of the cohort design. However, a relative (not absolute) estimate of test predictive performance may still be derived for people opting out of TPT, if two or more tests are evaluated simultaneously in the same cohort. Detailed guidance on how to conduct such studies is available in the framework for evaluation of tests for progression $[9,10]$.

Study design 2: sensitivity and specificity using clinical reference standards

Sensitivity can be assessed using culture-confirmed TB as a reference standard. This is important to reduce bias in the estimated accuracy. It should be encouraged even for paediatric TB and forms of

Study 1: predictive performance

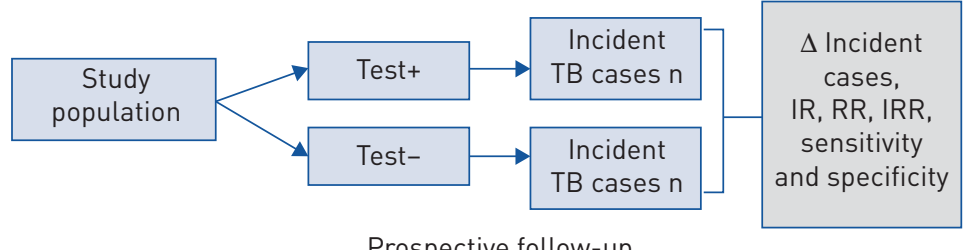

Prospective follow-up

Study 2: sensitivity and specificity
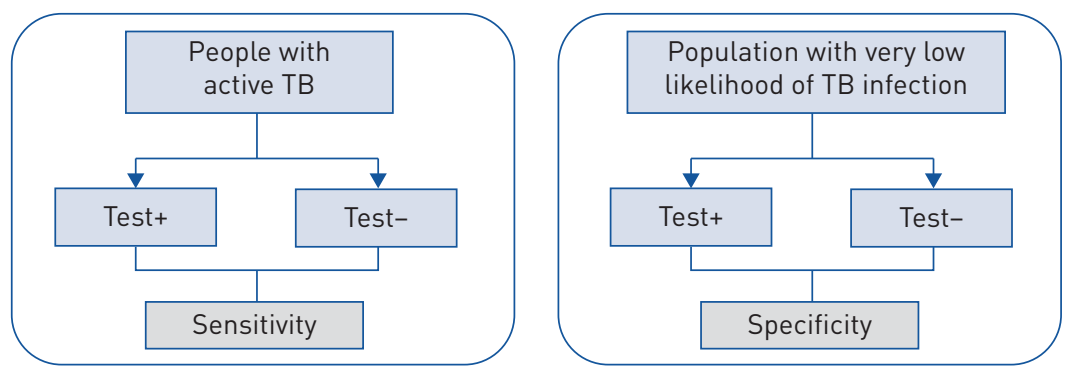

Study 3: concordance

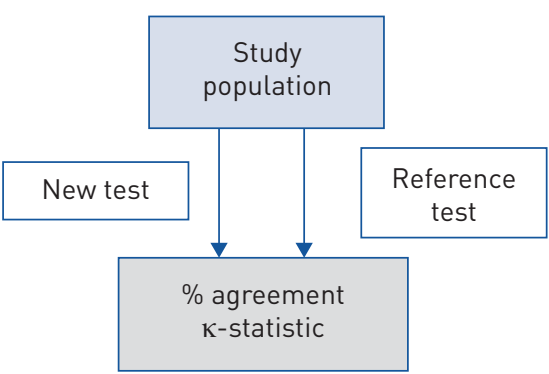

FIGURE 3 Study designs for the evaluation of tests for tuberculosis (TB) infection. Studies 1 and 2 include a single test in the figure for simplicity, but a new test should be compared with a reference test. Study design 1 is based on Kı et al. [9]; see therein for further details. IR: incidence rate; RR: risk ratio; IRR: incidence rate ratio. 
extrapulmonary TB that tend to be diagnosed clinically. A diagnosis based only on clinical or radiographic criteria should only be exceptional. In this case, it is important not to include tests for TB infection in the diagnostic criteria as this will spuriously overestimate the sensitivity of the test for infection.

Specificity depends on the antigens used to induce the immune response. By using overlapping peptides from antigens that are highly M. tuberculosis-specific, such as ESAT6 and CFP10, current IGRA tests are not affected by bacille Calmette-Guérin (BCG) vaccination, unlike TST, and their specificity is high at 97-99\% based on studies conducted in low-risk populations who are unlikely to have been infected with TB. However, expression of ESAT6 and CFP10 by a group of nontuberculous mycobacteria species, including $M$. marinum and $M$. kansasii, is well known to lead to false-positive test results in people infected with these species. For the estimation of specificity, the population is one with very low likelihood of prior exposure to $M$. tuberculosis. It is important to evaluate the impact of cross-reactions by conducting subgroup analyses by BCG vaccination status and by likelihood of exposure to nontuberculous mycobacteria; for example, based on geographical and demographic information [22]. For assessment of tests for TB infection based on TB-specific antigens not found in the BCG vaccine itself (such as ESAT6 or CFP10), vaccination status of participants does not need to be taken into consideration.

The tests should be performed at the same time as the clinical evaluation as a delay between them can introduce bias [22]. This can be part of a cohort study, but longitudinal follow-up is not essential. In addition, it is important to conduct the tests in different TB epidemiological settings, although specificity cannot be measured in settings with high prevalence of TB infection. As discussed earlier, estimation of sensitivity and specificity entails two different cohorts, people with active TB and individuals with very low likelihood of TB infection. Participants in each cohort should be selected randomly or consecutively when enrolled. It is particularly important to avoid selecting the study population from a larger group of potentially eligible people on the basis of clinical or disease characteristics that might affect test performance (although this is acceptable in early stages of the evaluation of new tests).

The tests should be performed by well-trained people experienced in the procedures for both the reference test and the new test. People performing and evaluating tests should be blinded to the results of the other tests. Methods for supervision, monitoring and quality control should be adequate and clearly described. These points also apply to studies of concordance.

\section{Study design 3: concordance of tests}

This design is essentially a study of agreement between new and reference tests and is the lowest level of evidence for assessment of diagnostic tests. It should be noted that concordance may be low when new tests are thought to have superior predictive performance or sensitivity and specificity. Therefore, this design is appropriate when the new test offers operational advantages over existing tests, but no gain in diagnostic performance is expected.

Study participants should be representative of the general population. For example, it is important to include very young or elderly people, pregnant women, people with more severe disease or people with serious comorbidities (e.g. HIV, diabetes, renal failure, malnutrition), as these may affect test performance. Studying agreement in different TB epidemiological settings and expected burdens of nontuberculous mycobacteria is encouraged.

When two tests are administered, they should be done at the same time, ideally on the same sample (if based on blood or urine). If analysing skin tests, the reference and index tests should be administered in each arm at the same time. Collection of blood or urine specimens for in vitro tests for TB infection should be done at the same time or before administering a TST to avoid a boosting effect [23].

Sample size calculation

Principles of sample size determination: superiority versus noninferiority

Superiority designs and related sample size calculations are appropriate if the reference test has suboptimal performance. For example, the sensitivity of current tests for TB infection is judged to be suboptimal. Sensitivity is particularly suboptimal in young children, PLHIV and otherwise immunocompromised people, who are at increased risk of disease and therefore a priority for testing for TB infection. Therefore, a new test would be of great interest if it had superior sensitivity, particularly in these high-risk populations.

If the reference test has excellent specificity, such as the 97-99\% specificity of current IGRAs, it would require a large sample size to demonstrate superiority (table 1) [24]. However, a new test could have 
TABLE 1 Example of changes in different key assumptions on sample size requirements to demonstrate superiority/noninferiority of tests for sensitivity and specificity

\begin{tabular}{|c|c|c|c|c|}
\hline & Reference & New test & Difference & Sample size required ${ }^{\#}$ ( $80 \%$ power) $n$ \\
\hline \multirow[t]{8}{*}{ Sensitivity } & 95 & 98 & 3 & 331 \\
\hline & 95 & 92 & 3 & 478 \\
\hline & 95 & 90 & 5 & 185 \\
\hline & 95 & 87 & 8 & 79 \\
\hline & 90 & 93 & 3 & 716 \\
\hline & 90 & 87 & 3 & 843 \\
\hline & 90 & 85 & 5 & 316 \\
\hline & 90 & 82 & 8 & 130 \\
\hline \multirow[t]{8}{*}{ Specificity } & 98 & 99 & 1 & 1283 \\
\hline & 98 & 95 & 3 & 233 \\
\hline & 98 & 93 & 5 & 96 \\
\hline & 98 & 90 & 8 & 44 \\
\hline & 95 & 98 & 3 & 331 \\
\hline & 95 & 92 & 3 & 478 \\
\hline & 95 & 90 & 5 & 185 \\
\hline & 95 & 87 & 8 & 79 \\
\hline
\end{tabular}

specificity that is noninferior to an existing test, and will be preferred because of other advantages. If a noninferiority design is selected, these other important advantages, such as lower cost to the patient or health system, enhanced feasibility, or point-of-care availability, must be pre-specified, and measured carefully. It is also important to note that noninferiority designs should be reserved to test performance when the standard or reference test has very good performance, such as the example of the specificity of the IGRA tests. This is necessary to avoid a risk for the progressive deterioration of test performance over time if newly emergent technologies are successively compared to the previous generation of test using noninferiority criteria [25].

\section{General determinants of sample size}

When designing a study, it is particularly important to pre-specify the effect size, or difference expected, for both superiority and noninferiority designs. In general, the larger the effect size, the smaller the sample size. It is important to recognise that larger effect sizes may not be realistic and may lead to erroneous conclusions. In a study with too few participants, a new test that has superior performance, but by a small difference from the reference test, may fail to show superiority due to a wide confidence interval crossing the null value. The estimates of performance of the reference test used for sample size calculation should be based on recently conducted high-quality systematic reviews to ensure their accuracy.

\section{Specific sample size calculations and study analysis}

\section{Predictive performance}

The new test would ideally have good sensitivity and reasonable specificity. However, new tests for TB infection are not expected to improve predictive value substantially, and most gains are expected to be seen in operational aspects. Hence, demonstrating noninferiority in terms of predictive performance would be acceptable.

Sample size calculations have to account for the likelihood of future TB disease, as this determines the number of events, the sensitivity of current tests in predicting these events, and differences in sensitivity of the new test and follow-up time. While longer periods of follow-up reduce the required sample size, it may result in greater losses to follow-up and lead to higher risk of reinfection in high-transmission settings, which could complicate interpretation of the initial test. Hence, longer periods of follow-up are not encouraged, at least for the primary analysis and sample size calculations.

The event rate in cohort studies is typically calculated as the number of events per 100 person-years of follow-up, which accounts for variable follow-up times in a large-scale cohort. Since the same people will have had two or more tests for TB infection, the differences in event rates can be directly calculated, either 
as a risk difference or as a risk ratio. The incidence rate ratio can be estimated as incidence rate among people who test positive/incidence rate among people who test negative.

\section{Sensitivity and specificity}

Current tests for TB infection generally have suboptimal sensitivity. Sensitivity is lower in children, PLHIV and other immunocompromised people. In these populations, superiority of a new test would be preferable to a noninferiority design.

Current IGRA tests have excellent specificity. Therefore, superior specificity is neither necessary nor likely to be demonstrable. Hence, a noninferiority design is sufficient for specificity when the reference standard test is an IGRA, or a TST in a population that has not been BCG-vaccinated. If the reference standard is TST in a BCG-vaccinated population, then a superiority design is recommended.

Sensitivity and specificity can be calculated using the standard formula based on the aforementioned reference standards. However, in a population with very low TB prevalence, specificity can be approximated using the total number of people tested as the denominator instead of the number of people without TB infection, as follows: all people who test negative/all people tested in the very-low-prevalence population.

\section{Concordance}

If the new test is anticipated to have similar diagnostic accuracy to the reference test, but has operational advantages, such as lower cost or greater feasibility, then tests showing high agreement are valuable. The $\kappa$-statistics should be calculated, with the accompanying 95\% confidence interval. $\kappa$-statistics account for chance-corrected agreement, which is especially important when prevalence of positive tests is either very low or very high. The sample size is determined by the maximum acceptable width of the $\kappa 95 \%$ confidence interval, the underlying true proportion of positives and the anticipated value of $\kappa$ [26].

\section{Technical issues}

Immunoassays are complex assays influenced by multiple sources of variability which can impact results. Table 2 lists a range of typical sources of variability in in vitro tests for TB infection such as IGRA, which should be prioritised by developers in the technical description of the assay. A full list is available elsewhere [16].

As immunoassays detect responses on a continuous scale, which is converted to a binary outcome as positive or negative by use of a threshold value (cut-off), a description of the variability around this cut-off is of particular relevance. To determine the degree of variability around the cut-off threshold of any new test, we recommend to evaluate changes in IGRA results by using an adequate number of participants without active TB with positive and negative IGRA values representative of normal physiological ranges in cohorts where prior infection is likely and reinfection events are rare. For example, studies in recent adult migrants from high TB incidence countries to low-to-middle incidence countries where TPT is not routinely initiated for a positive IGRA result could examine reproducibility of the initial IGRA positivity with minimal influence from reinfection. Similarly, reproducibility of IGRA results has often been evaluated in healthcare workers, demonstrating fluctuation of results [27]. Contacts of people with TB who recently converted to a

TABLE 2 Typical sources of variability in interferon- $\gamma$ release assay-like tests items for documentation

Factors impacting stimulation assay

Analytical range of readout assay
Imprecision of the readout assay
Accuracy of readout assay
Analytical specificity of readout assa

Additional assessments
Blood collection tubes: within- and between-lot variability

Delay in blood processing and incubation time

Volume of blood

Limit of detection

Lower limit of quantification

Intra-assay and inter-assay imprecision, in particular around the cut-off for test positivity Recovery

Cross-reactivity

Parallelism/dilution linearity

Common interferents (e.g. rheumatoid factor, lipids, bilirubin, complement, haemolysate)

Evaluation of curve-fitting model ( $\geqslant 5$ determinations over multiple runs) Inter-laboratory imprecision (reproducibility)

Analyte stability (freeze-thaw stability, short-term bench stability, long-term storage stability)

LoD: limit of detection; LLoQ: lower limit of quantification. 
positive test could also be followed-up to assess possibility of reversion. Samples should be used to estimate the rate of IGRA conversions/reversions using the predefined cut-off for assay positivity. In cases of IGRA conversion, a third sample may be collected to evaluate if conversion using the pre-defined cut-off was sustained due to an $M$. tuberculosis infection event, rather than a spurious effect of variability around the cut-off. Samples from such participants should be excluded when defining the range of the zone of uncertainty.

The QuantiFERON-TB Gold In-Tube and QuantiFERON-TB Gold Plus assays report considerable variability around the assay cut-off of $0.35 \mathrm{IU} \cdot \mathrm{mL}^{-1}$ [28]. While the cut-off value for any new IGRA should be defined against the concordance with existing tests such as QuantiFERON-TB Gold Plus, manufacturers should be cautious about reporting test results around this zone of uncertainty. Assessment of any new IGRA should also report IU.mL ${ }^{-1}$ for the mitogen, the antigen and the unstimulated control separately. Each new IGRA will need to study zone of uncertainty. Assays with only a binary readout for TB infection should provide additional data to confirm reproducibility without resulting in a high number of invalid results. Participant samples from both populations with very low likelihood of TB infection as well as people with confirmed TB disease should be used to establish a range of interferon- $\gamma$ responses expected for clinically relevant specimens.

\section{Evaluation of safety for skin tests}

Safety of new skin tests should be evaluated against a reference skin test (TST) in a population representative for the target population for the new test to show that injection site reactions and other adverse events are similar to or fewer than with TST. Safety should be evaluated in various groups such as PLHIV, children and pregnant and lactating women. TST is safe to administer to pregnant women and lactating women. Similarly, it is unlikely that new skin tests for TB infection cause adverse effects on the fetus or nursing infants when administered to pregnant or lactating women.

The study design should seek to minimise bias in ascertaining local adverse reactions. One method is to give the new and the reference test at the same time in each forearm in a double-blinded manner. Another method to assess safety would be to compare adverse events reporting in a randomised controlled trial. However, trials are unlikely to be sufficient to detect rare adverse events and post-marketing surveillance is essential. Methods for recording adverse events should be adequate and clearly recorded, e.g. using MedDRA classification (www.meddra.org/). As skin tests are designed to induce a local reaction, it is important to pre-define how to interpret reactions as relevant indurations or adverse events.

\section{Economic evaluation}

New tests should ideally have lower health system and patient costs compared to existing tests. In order to evaluate this aspect, costs associated with both the start-up and routine operations of the new test should be considered in studies that are evaluating the new test. Ideally, an economic evaluation should be incorporated into studies run at demonstration sites or independent research studies. Regardless of the study setting, it is important that an effort is made to document the "true" cost of implementing the new test (i.e. as it would be used in practice). The following costs should be considered: laboratory equipment and start-up; computers and software; supplies; cold-chain requirements; personnel time for different aspects of testing; initial and ongoing training; quality control and supervision; and health facility visits by patients with positive or negative tests. Optimum care organisation models should be used when implementation is being considered (e.g. "one-stop shop" where follow-up care for those with positive tests is coordinated and provided same day). Additional details on specific costs to consider during evaluations are available presented in the supplementary table.

\section{Operational characteristics}

New tests for TB infection should also address operational challenges associated with the existing tests. The ability to deploy at the lowest level of the healthcare system is especially important. Instrument-free tests or tests that can be performed with a small, portable or hand-held instrument that function by battery or solar power are needed. Rapid tests (e.g. $<1 \mathrm{~h}$ for results) would also offer a great advantage. For both skin tests and in vitro tests, the stability of reagents should be established under different conditions in accordance with the WHO standards for pre-qualification [29]. It is desirable that reagents are stable at high temperature and humidity for sufficient periods and that a cold chain is not required for their transportation. Tests that do not require the withdrawal of venous blood samples would be preferred.

\section{Concluding remarks}

The uptake of TPT has been very slow globally, partly due to concerns about performance and operational challenges associated with current tests. New tests for TB infection offer an opportunity to facilitate the 
scale-up and targeting of TPT for more effective use. They also support other initiatives to improve TB preventive treatment, such as the new target product profiles released in 2020. Implementation of this framework for the evaluation of new tests for TB infection will facilitate standardised evaluation of new tests and will expedite their adoption into national policies at the scale needed to achieve global targets.

Acknowledgements: We thank the following individuals for their critical review of the original document published by WHO: Sevim Ahmedov (United States Agency for International Development, USA), Claudia Denkinger (University of Heidelberg, Germany), Darragh Duffy (Institut Pasteur, France), Nazir Ismail (National Institute for Communicable Diseases, South Africa), Afrânio Kritski (Federal University of Rio de Janeiro, Brazil), Elisa Nemes (University of Cape Town, South Africa) and Molebogeng X. Rangaka (University College London, UK).

Conflict of interest: Y. Hamada has nothing to disclose. S. den Boon has nothing to disclose. D.M. Cirillo has nothing to disclose. A. Penn-Nicholson: FIND has several clinical research projects to evaluate multiple new diagnostic tests against published target product profiles that have been defined through consensus processes. These studies are for diagnostic products developed by private sector companies who provide access to know-how, equipment/reagents, and contribute through unrestricted donations as per FIND policy and external SAC review. FIND has not allocated any financial value to know-how or access to equipment gained through these projects. Current projects are listed at www.finddx.org for more information. In addition, A. Penn-Nicholson has a patent WO2016123058A1 WIPO (PCT) (Biomarkers for detection of tuberculosis risk 2016-08-04) pending, and a patent WO2017081618A9 WIPO (PCT) (Biomarkers for prospective determination of risk for development of active tuberculosis 2017-07-13) pending. M. Ruhwald: FIND has several clinical research projects to evaluate multiple new diagnostic tests against published target product profiles that have been defined through consensus processes. These studies are for diagnostic products developed by private sector companies who provide access to know-how, equipment/reagents, and contribute through unrestricted donations as per FIND policy and external SAC review. FIND has not allocated any financial value to know-how or access to equipment gained through these projects. Current projects are listed at www.finddx.org for more information. In addition, M. Ruhwald has a patent W02017084671A1 issued to Statens Serum Institute, and a patent 02008028489A2 issued to Statens Serum Institute. D. Menzies has nothing to disclose. O. Oxlade has nothing to disclose. D. Falzon has nothing to disclose. A. Kanchar has nothing to disclose. A. Korobitsyn has nothing to disclose. M. Zignol has nothing to disclose. A. Matteelli has nothing to disclose. T. Kasaeva has nothing to disclose.

\section{References}

$1 \quad$ World Health Organization (WHO). Implementing the End TB Strategy: The Essentials. Geneva, WHO, 2015.

2 Lönnroth K, Migliori GB, Abubakar I, et al. Towards tuberculosis elimination: an action framework for low-incidence countries. Eur Respir J 2015; 45: 928-952.

3 United Nations General Assembly. A/RES/73/3. Political declaration of the High-level Meeting of the General Assembly on the Fight Against Tuberculosis: resolution/adopted by the General Assembly. New York, United Nations General Assembly, 2018. http://digitallibrary.un.org/record/1649568

4 World Health Organization. Global Tuberculosis Report 2020. Geneva, WHO, 2020. www.who.int/publications/ i/item/9789240013131

5 Getahun H, Matteelli A, Chaisson RE, et al. Latent Mycobacterium tuberculosis infection. N Engl J Med 2015; 372: $2127-2135$.

6 World Health Organization (WHO). WHO Consolidated Guidelines on Tuberculosis: Module 1: Prevention: Tuberculosis Preventive Treatment. Geneva, WHO, 2020.

7 Rangaka MX, Wilkinson KA, Glynn JR, et al. Predictive value of interferon- $\gamma$ release assays for incident active tuberculosis: a systematic review and meta-analysis. Lancet Infect Dis 2012; 12: 45-55.

8 Matteelli A, Sulis G, Capone S, et al. Tuberculosis elimination and the challenge of latent tuberculosis. Presse Med 2017; 46: e13-e21.

9 Kik SV, Schumacher S, Cirillo DM, et al. An evaluation framework for new tests that predict progression from tuberculosis infection to clinical disease. Eur Respir J 2018; 52: 1800946.

10 World Health Organization (WHO). Consensus Meeting Report: Development of a Target Product Profile (TPP) and a Framework for Evaluation for a Test for Predicting Progression from Tuberculosis Infection to Active Disease. Geneva, WHO, 2017.

11 World Health Organization (WHO). Use of Tuberculosis Interferon-Gamma Release Assays (IGRAs) in Low- and Middle-Income Countries Policy Statement. Geneva, WHO, 2011.

12 Slogotskaya L. Sensitivity of a novel skin test with recombinant protein ESAT6-CFP10 in new cases of tuberculosis in children and adolescents. Pediatr Pulmonol 2014; 49: S65-S66.

13 Li F, Xu M, Qin C, et al. Recombinant fusion ESAT6-CFP10 immunogen as a skin test reagent for tuberculosis diagnosis: an open-label, randomized, two-centre phase 2a clinical trial. Clin Microbiol Infect 2016; 22: 889-889. 
14 Ruhwald M, Aggerbeck H, Gallardo RV, et al. Safety and efficacy of the C-Tb skin test to diagnose Mycobacterium tuberculosis infection, compared with an interferon $\gamma$ release assay and the tuberculin skin test: a phase 3, double-blind, randomised, controlled trial. Lancet Respir Med 2017; 5: 259-268.

15 Aggerbeck H, Ruhwald M, Hoff ST, et al. C-Tb skin test to diagnose Mycobacterium tuberculosis infection in children and HIV-infected adults: a phase 3 trial. PLoS One 2018; 13: e0204554.

16 World Health Organization. Framework for the Evaluation of New Tests for Tuberculosis Infection. Geneva, WHO, 2020.

17 Hamada Y. State of the art: tests for latent TB infection. AIDS 2020 virtual pre-conference: Turning threats into opportunities: implementing and advancing quality TB services for people living with HIV during the COVID-19 pandemic and beyond. https://www.iasociety.org/Web/WebContent/File/TBHIV-2020-Programme26Jun2020.pdf

18 Lu P, Chen X, Zhu L, et al. Interferon-gamma release assays for the diagnosis of tuberculosis: a systematic review and meta-analysis. Lung 2016; 194: 447-458.

19 Nasiri MJ, Pormohammad A, Goudarzi H, et al. Latent tuberculosis infection in transplant candidates: a systematic review and meta-analysis on TST and IGRA. Infection 2019; 47: 353-361.

20 Barcellini L, Borroni E, Brown J, et al. First evaluation of QuantiFERON-TB Gold Plus performance in contact screening. Eur Respir J 2016; 48: 1411-1419.

21 Getahun H, Matteelli A, Abubakar I, et al. Management of latent Mycobacterium tuberculosis infection: WHO guidelines for low tuberculosis burden countries. Eur Respir J 2015; 46: 1563-1576.

22 Honda JR, Virdi R, Chan ED. Global environmental nontuberculous mycobacteria and their contemporaneous man-made and natural niches. Front Microbiol 2018; 9: 2029.

23 van Zyl-Smit RN, Pai M, Peprah K, et al. Within-subject variability and boosting of T-cell interferon- $\gamma$ responses after tuberculin skin testing. Am J Respir Crit Care Med 2009; 180: 49-58.

24 Sotgiu G, Saderi L, Petruccioli E, et al. QuantiFERON TB Gold Plus for the diagnosis of tuberculosis: a systematic review and meta-analysis. J Infect 2019; 79: 444-453.

25 Everson-Stewart S, Emerson SS. Bio-creep in non-inferiority clinical trials. Stat Med 2010; 29: 2769-2780.

26 Watson PF, Petrie A. Method agreement analysis: a review of correct methodology. Theriogenology 2010; 73 : 1167-1179.

27 Joshi M, Monson TP, Joshi A, et al. IFN- $\gamma$ release assay conversions and reversions. Challenges with serial testing in U.S. health care workers. Ann Am Thorac Soc 2014; 11: 296-302.

28 Nemes E, Rozot V, Geldenhuys H, et al. Optimization and interpretation of serial QuantiFERON testing to measure acquisition of Mycobacterium tuberculosis infection. Am J Respir Crit Care Med 2017; 196: 638-648.

29 World Health Organization (WHO). Technical Guidance Series for WHO Prequalification - Diagnostic Assessment: Establishing Stability of in vitro Diagnostic Medical Devices. Geneva, WHO, 2017. 